\title{
ROTATION JOINTS FRICTION IN A TRANSVERSAL MOBILE COUPLING
}

\author{
Cornel Catalin Gavrila, Mihai Lates \\ “Transilvania” University of Brasov, Product Design Faculty, Mechatronics and Environment Department, \\ Brasov, Romania
}

Corresponding author: Cornel Catalin Gavrila, cgavrila@unitbv.ro

\begin{abstract}
The transversal mobile couplings are used in movement and torque transmission between two main shafts having parallel axis, with the possibility to undertake misalignments in the transversal plane. These shaft misalignments are usually named transversal movements. The paper presents, starting from some kinematic aspects regarding relative movements between the coupling parts, the kinematic equations useful in determining position of the intermediary elements, depending by the transversal misalignments. Following this, using the inside relative movement in rotation joints between parts and corresponding adequate materials, the friction coefficient is studied. The friction which appears in coupling's rotation joints between the involved parts has an important influence on their dynamic behaviour, wear and lifetime. The most significant friction and also wear is given by the alternant rotation movements in joints between parts, at the ends of their angular stroke. Due to this, the study of the friction between some materials to be used in coupling manufacturing is required, but also difficult because of some particularities, as reduced rotation in joints, for reduced transversal misalignments between the main shafts. There are presented, in the paper final part, the results and conclusions.
\end{abstract}

Key words: Mobile coupling, transversal misalignments, rotation joints, angular stroke, friction

\section{INTRODUCTION}

Generally, the mobile couplings are used in movement and torque mechanical transmission, between two shafts with various axial, radial or angular misalignments. Particularly, the mobile transversal couplings are designed to be used between the shafts with parallel axis, radial misaligned $[1,2]$. Inside the transversal mobile coupling, the shafts are connected by various kinematical linkages which allow the translations in the transversal plane, named transversal movements or eccentricities. Depending by the coupling type, the inside linkage can be structured by having translation joints, rotation joints or both translation and rotation joints. Also, in operation, this coupling linkage is needed to transmit the same angular velocity between shafts. The mechanisms theory indicates that, if the shafts are connected with joints to the basis, it will result the coupling corresponding associated mechanism, which is a plane mechanism with translation joints, rotation or both combined [1-3].

One of the simplest known couplings that may undertake a transversal movement between two semi couplings, each assembled on rotational shaft, is the Semiflex coupling $[1,2]$. As structure, this coupling type consists in three main elements: the input semi coupling, the intermediary element and the output semi coupling, as is presented in Figure 1. Between them, there are linkages with rotation joints disposed on two parallelogram contours: one between the input semi coupling 1 and the intermediary element 2 , the other, between the intermediary element 2 and the output semi coupling 3. The parallelogram contours are oriented each other particularly at 90 degrees $[1,2]$. Due to the mobile coupling dynamic behaviour, at lower speed the undertaken eccentricity is largely increased [4].

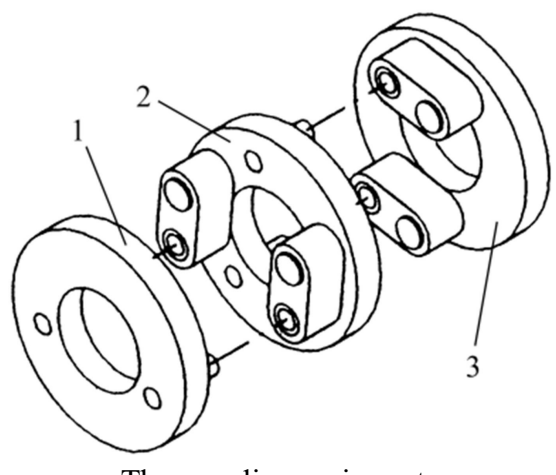

The coupling main parts

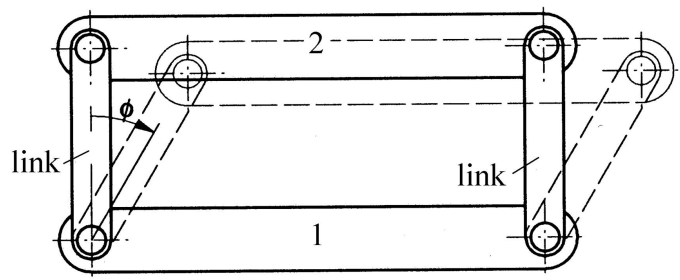

The parallellogram contour Fig. 1. The Semiflex coupling $[1,2]$ 
The main inconvenient of this mobile coupling type is the friction inside the rotation joints between the link and semi coupling or intermediary element bolt. Many producers are focused to reduce the frictional loses between elements. A solution to reduce the friction inside coupling is to lubricate the joints, but in some situation there are some inconvenient, as coupling operating temperature or environmental contaminations. As constructive solutions to reduce friction, there are placed some bearings inside joints (which can led to increased dimension), or the metal links are replaced with plastic parts $[1,4,5]$.

In Figure 2, there are presented two examples of modern Semiflex type mobile couplings, a constructive normal version and also a compact one, having reduced dimensions to be used in extreme limited space [4- 6].

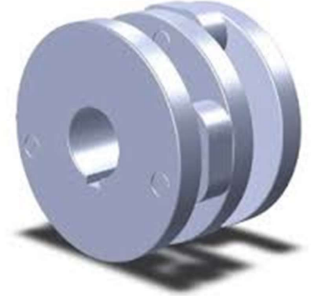

Normal version

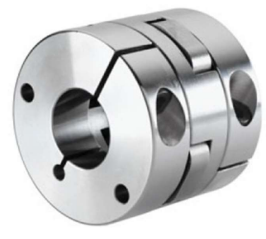

Compact version
Fig. 2. Semiflex coupling constructive examples $[4,5]$.

\section{THE COUPLING KINEMATICAL MODEL}

For the studied coupling from in Figure 1, the geometrical and kinematical model is presented in Figure 3. In this model, the parts are defined in the reference system $O_{i} x_{i} y_{i}$. Also, for each reference system the origin is considered in the gravity centre, $G_{i}$ and the reference axis orientation is convenient chosen.

To define the geometrical restrictions, is necessary to know the point's coordinates, relative to the local coordinate system and also relative to the fixed coordinates system. The general relation for these coordinates is [2]:

$$
\left[\begin{array}{l}
x_{M_{i}} \\
y_{M_{i}}
\end{array}\right]=\left[\begin{array}{l}
x_{0 i} \\
y_{0 i}
\end{array}\right]+\left[\begin{array}{cc}
\cos \varphi_{i}-\sin \varphi_{i} \\
\sin \varphi_{i} & \cos \varphi_{i}
\end{array}\right]\left[\begin{array}{l}
r_{i x} \\
r_{i j}
\end{array}\right],
$$

for $i=1,2,3$.

To define the rotation between two bodies, $i$ and $j$, the general condition is $P_{i} \equiv P_{j}$. The considered rotation appears between the input semi coupling and fixed reference element and, respectively, the output semi coupling and fixed reference element; from relation (1) result

respectively

$$
x_{\mathrm{o} 1}=0, y_{\mathrm{o} 1}=0
$$

$$
x_{03}=e_{x}, y_{03}=e_{y} .
$$

$$
e^{2}=e_{x}^{2}+e_{y}^{2}
$$

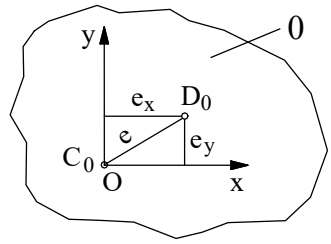

Reference element, 0

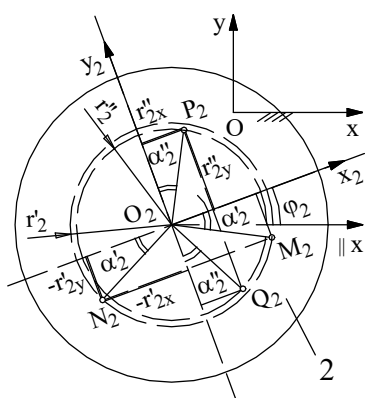

Intermediary element, 2

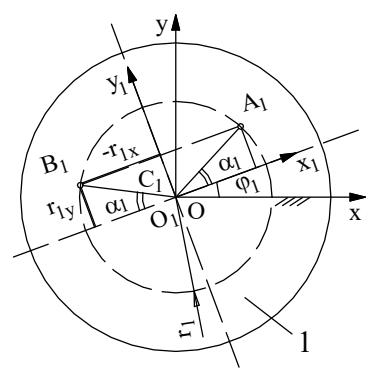

Input semicoupling, 1

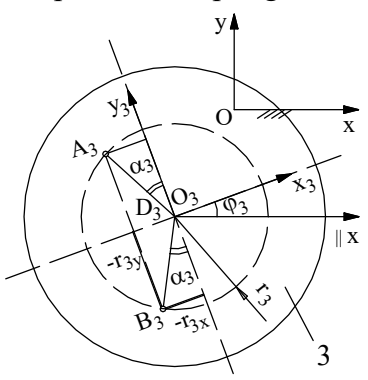

Output semicoupling, 3
Fig. 3. The coupling kinematical model

For the linkages with rotation joints disposed on parallelogram contours between two bodies $i$ and $j$, the general condition is:

$$
\left(x_{P_{j}}-x_{P_{i}}\right)^{2}+\left(y_{P_{j}}-y_{P_{i}}\right)^{2}=l^{2}
$$

The kinematic restriction equation is

$$
\rho_{1}-f(t)=0 \text {. }
$$

Based on the previously relations result:

and

$$
\mathrm{r}_{2}^{\prime} \cos \alpha_{2}^{\prime}=\mathrm{r}_{1} \cos \alpha_{1}
$$

$$
\mathrm{r}_{2}^{\prime \prime} \cos \alpha_{2}^{\prime \prime}=\mathrm{r}_{3} \cos \alpha_{3}
$$

Taking account by the links parallelism and length, from relation (7) result $\varphi_{1}=\varphi_{2}+2 K \pi$, where $K \in Z$, for $\forall \varphi_{1}, \varphi_{2} \in R$. For $K=0$, result $\varphi_{2}=\varphi_{1}$.

Also, from relation (8), result $\varphi_{2}=\varphi_{3}+2 K \pi$, where $K \in Z$, for $\forall \varphi_{2}, \varphi_{3} \in R$. As previously, $K=0$ and result is $\varphi_{2}=\varphi_{3}$, which led to the condition

$$
\varphi_{3}=\varphi_{1}
$$

From relation (9) it can be concluded that the angular velocity between shafts is the same.

From Figure 3, result the eccentricity relation 
As well, for given coupling dimension, the alternant relative motion inside the rotation joints between parts $i$ and $j$ are influenced only by the eccentricity amplitude $e$, as represented in Figure 4. The general relation is

$$
\Phi_{i, j}=f(e)
$$

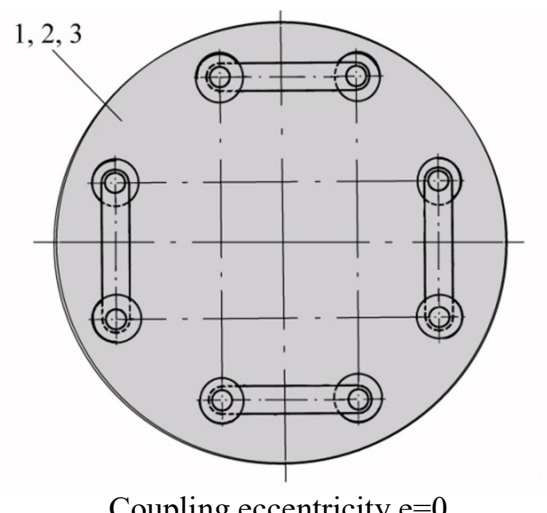

Coupling eccentricity e $=0$

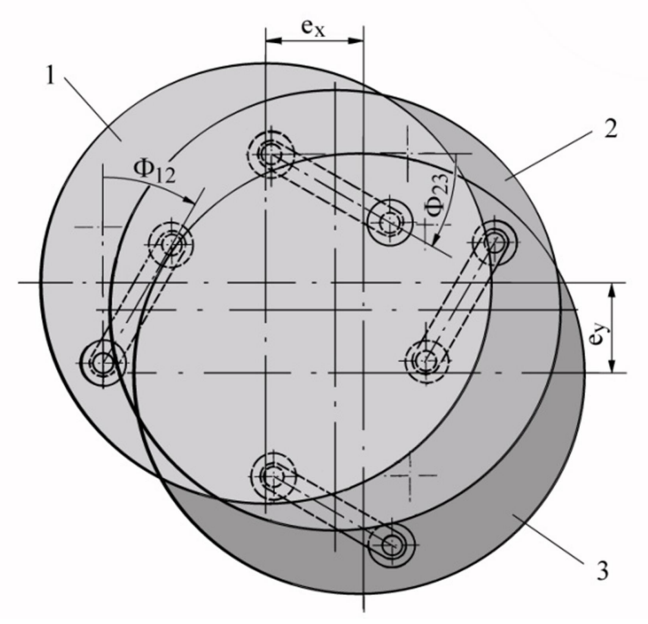

Coupling eccentricity e $\neq 0$

Fig. 4. The coupling eccentricity and relative motion in the rotation joints

Taking account by previous relations it can be observed that, during one coupling complete rotation, each parallelogram contour passes two times through a particular position when the eccentricity direction coincides with the parallel sides between the semi coupling and intermediary element. On this position, the eccentricity has the maximum value only along $x$ direction while on $y$ axis is 0 , respectively; the eccentricity has the maximum value only along $y$ direction while on $x$ axis is 0 . Because of the both parallelogram contours displacement, particularly at 90 degrees, these particular eccentricity undertake appears four times on a complete coupling rotation. Taking account on the coupling rotation joint functioning condition, the literature indicates that the alternant rotational movement variation in joints has the same periodicity $[2,7,8]$.

\section{THE FRICTION IN THE ROTATION JOINTS}

To achieve the friction tests inside the mobile coupling joints, is used a test rig which has as components a tribometer and a computer used for the acquisition of the measured data $[9,10]$. The tribometer used has a force sensor placed on two sliders, for vertical motions, with a stroke up to 75 $\mathrm{mm}$ and a speed between 0.01 and $10 \mathrm{~m} / \mathrm{s}$. The sensor also allows the forces measurements along the vertical and also, a horizontal direction, in the interval of 0.1 to $1000 \mathrm{~N}$, with the resolution of 50 $\mu \mathrm{N}$. For the lateral position, resolution is $\mathrm{R}_{\mathrm{r}}=2 \mu \mathrm{m}$. The oscillatory motion module, presented in Figure 5, allow the alternant rotational oscillating motion. To complete the testing and acquisition data system, the tribometer is connected to an acquisition plate, to a computer.
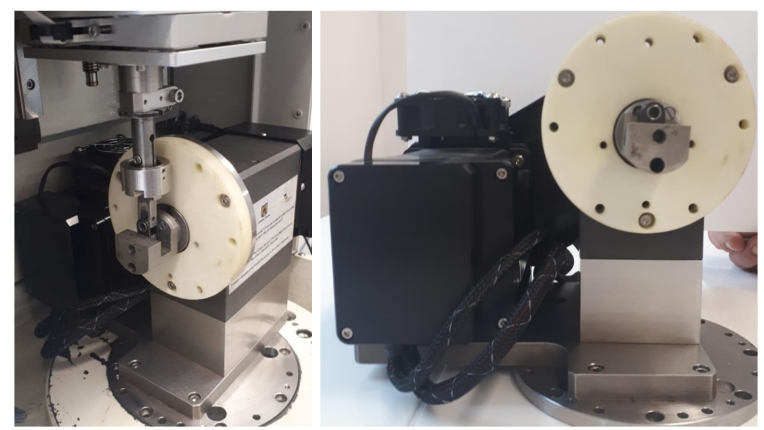

Fig. 5. The test rig and the oscillatory motion module

For testing the subjected rotation joint pieces (link and bolt), it was used adequate device to keep these small parts in relative alternant motion. The bolt is adjusted in the centre of its alternant rotating device, assembled in the tribometer adequate oscillatory motion module, as is presented in Figure 5. To realise the rotation joint, the bolt has also to fit into the link attached thru a holder, as in real functioning conditions.

To estimate the friction inside the coupling rotation joints, both coupling joint parts correspond to a standard Semiflex mobile coupling, size F 320 [4], made from medium-carbon steel, not greased. According to the coupling rotation joint functioning condition [7], the tests are performed for the alternant rotational motion, in the interval from \pm 5 to \pm 25 degrees, at an angular speed of $5 \mathrm{rpm}$. The load normal force is variable between 10 to $50 \mathrm{~N}$.

In Figure 6 is presented the variation of the friction coefficient during the motion. Along the motion corresponding portion of the curve, the dynamic friction coefficient can be observed. At the ends of the angular stroke in joint, it can be observed the increased values of the static friction coefficient. The situation corresponds to the particular position when 
the alternant rotational motion in joints changes the sense.

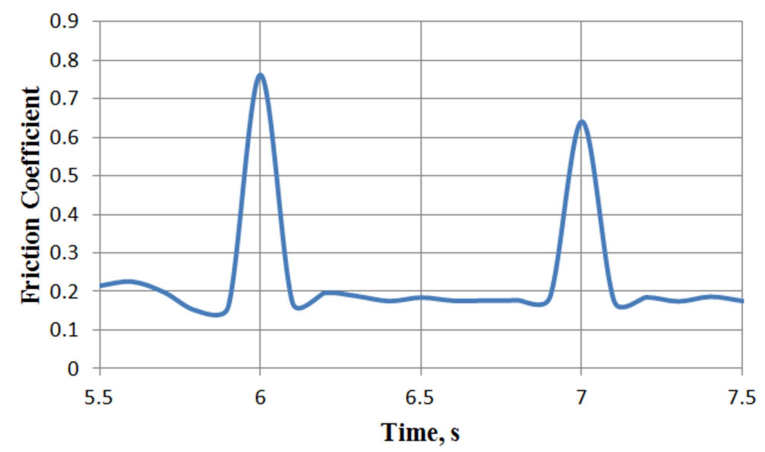

Fig. 6. The variation of the friction coefficient during the motion

In Figure 7, the dynamic friction coefficient variation depending by the load force, from 10 to $50 \mathrm{~N}$, is presented. If the load forces increase, the dynamic friction coefficient value is slightly increasing from 0.2 to 0.27 .

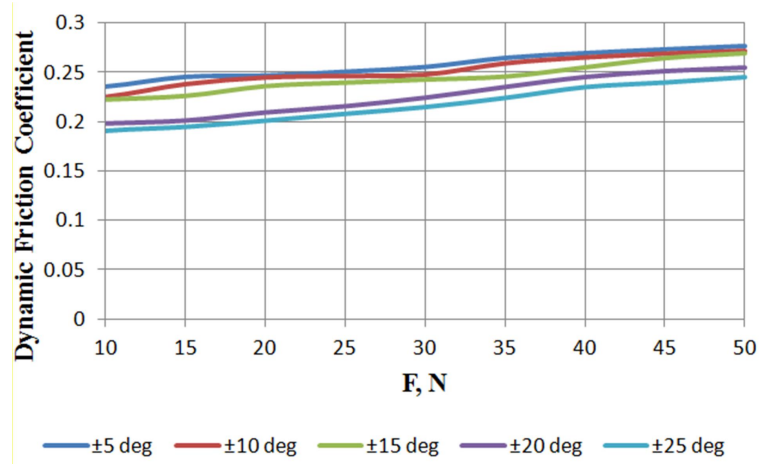

Fig. 7. The dynamic friction coefficient depending by the load force

In Figure 8, the static friction coefficient is presented, for the same loads values. It can be observed that, for different angular stroke values, the static friction coefficient value is around 0.65 .

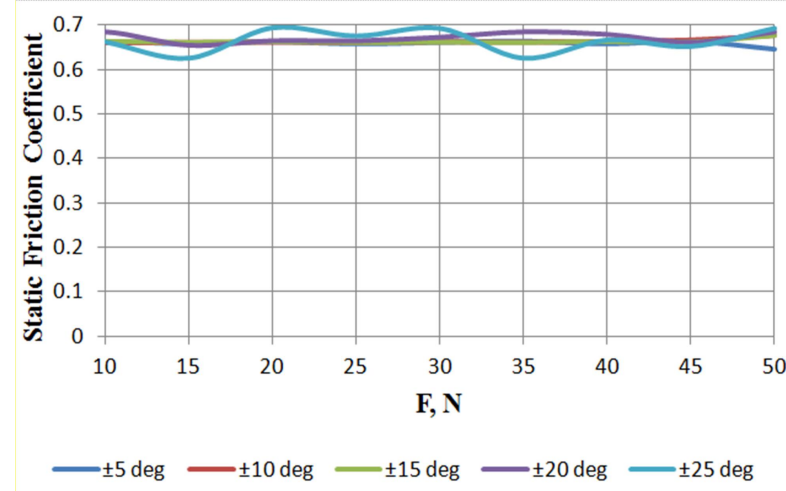

Fig. 8. The static friction coefficient depending by the load force
In Figure 9, the dynamic friction coefficient variation depending by the different angular stroke, from $\pm 5^{\circ}$ to $\pm 25^{\circ}$, is presented. If the angular stroke increases, the dynamic friction coefficient value is slightly decreasing.

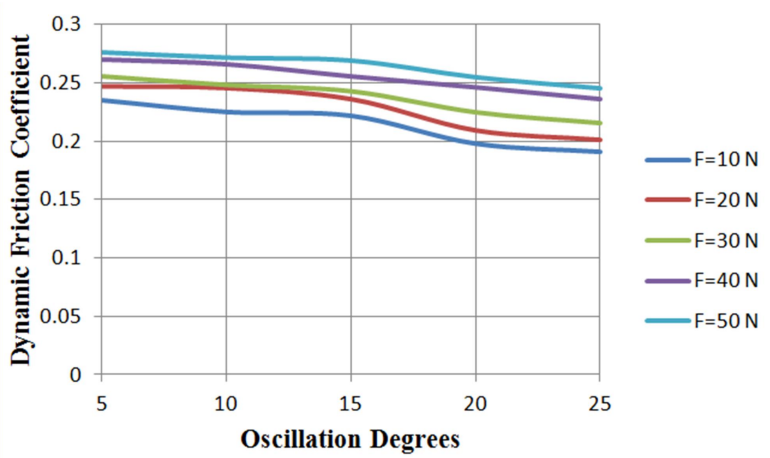

Fig. 9. The dynamic friction coefficient depending by the angular stroke

In Figure 10, the static friction coefficient is presented, for the same angular stroke values. For different applied load force values, the friction dynamic coefficient value is also around 0.65 .

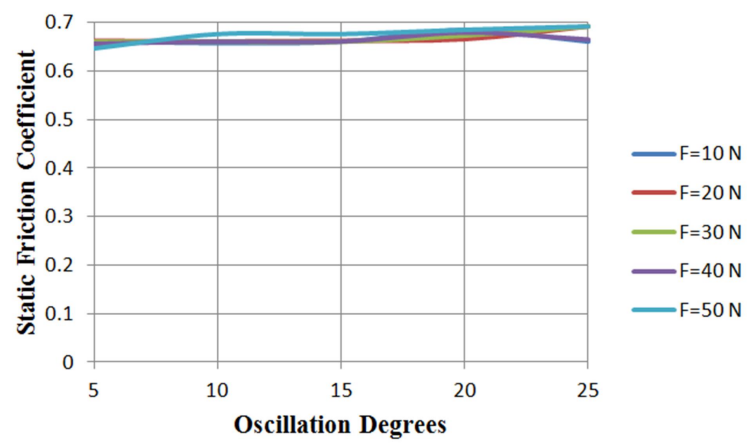

Fig. 10. The static friction coefficient depending by the angular stroke

\section{CONCLUSIONS}

During the alternant oscillatory motions, the contact between the tested link and pin rotation joint parts is characterised by two types of friction coefficients: the static friction coefficient - which appears at the ends of the angular stroke, when the motion changes the direction, and also the dynamic friction coefficient during the proper alternant motion).

The static friction coefficient inside the rotation joints appears in coupling functioning conditions when the alternant movement is changing their direction; each rotation joint friction changes the direction twice on a coupling rotation. For decreased load force and reduced angular stroke, the static coefficient value is close to 0.65 . If the angular stroke increase at the same applied force, the static friction coefficient slightly increases up to 0.7 . 
The dynamic friction coefficient in these joints appears when the movement is maintaining the rotation sense; the size of these angular strokes depends by the coupling eccentricity. An increased eccentricity led to increased angular strokes in rotation joints and a slightly decreased dynamic friction coefficient, around 0.2. If the applied force increase, the dynamic friction coefficient slightly increases up to around 0.27 .

For a given transversal mobile coupling type Semiflex, by the friction in joint point of view, the better behaviour during functioning is obtained when the eccentricity between shafts is increased.

\section{REFERENCES}

1. Dudiţă Fl et al (2001), Articulated Mobile Couplings. Ed. Orientul Latin Braşov (in romanian).

2. Gavrilă C C (2009), Comparative Kinematics of Mobile Transversal Coupling, As Multibody System. Springer Science + Business Media B.V. pp $1-8$.

3. Shigley J E (1959), Kinematic Analysis of Mechanisms. McGraw-Hill New York.

4. *** Semiflex Schmidt-Kupplung GmbH 10/2015.

5. *** Schmidt Semiflex Shaft Couplings ABSSAC Ltd august 2013.

6. https://www.schmidt-

kupplung.com/en/products/semiflex.html, accessed in 08.02.2021.

7. Gavrilă C C, (2013), A Transversal Coupling $3 D$ Modeling Proceedings of the Annual Session of Scientific Papers XII pp 133-136.

8. Haarmann et. al., (1994), Lageberechnung bei querbeweglichen Wellenkupplungen, Antriebstechnik, 7 pp $47-51$ (in german).

9. M T Lates et al., (2016) Sliding friction study of the oscillating translational motion for steel on PA66 and PA46 type materials, IOP Conf. Ser.: Mater. Sci. Eng. 147012038 pp $1-5$.

10. *** (2009), UMT Multi-Specimen Test System. Hardware Installation \& Application Manual, Center for Tribology Dell Ave Campbell, USA.
Received: April 17, 2021 / Accepted: December 20, 2021 / Paper available online: December 25, 2021 (C) International Journal of Modern Manufacturing Technologies 